\title{
Qualité de l'insertion socioprofessionnelle de nouveaux travailleurs et travailleuses diplômés: Examen d'un schéma exploratoire*
}

\section{GENEVIÈVE FOURNIER, CHANTALE JEANRIE, \& LINE CROTEAU}

Université Laval

\section{RÉSUMÉ}

Si les enjeux liés à l'insertion socioprofessionnelle s'étendent bien au-delà des données factuelles et sont aussi influencés par des données inscrites dans la réalité subjective de l'individu, il est important d'identifier ces données qui permettent justement de prédire la qualité de l'insertion de nouveaux travailleurs et des nouvelles travailleuses. L'objectif de l'article est de présenter un schéma exploratoire regroupant trois catégories de variables définissant la qualité de l'insertion socioprofessionnelle et trois autres catégories de variables susceptibles de prédire la qualité de cette insertion. Ce schéma a été mis à l'épreuve auprès d'un sous-échantillon de 97 nouveaux travailleurs et des nouvelles travailleuses diplômés de programmes d'études post-secondaires. Les résultats des analyses de régression mettent en lumière la force relative de certains critères objectifs et subjectifs indiquent que toutes les variables indépendantes du schéma, sauf l'âge, permettent de saisir les facettes de la qualité de l'insertion des jeunes diplômés. ${ }^{1}$ Trois constatations

* Cette recherche a été réalisée grâce à une subvention octroyée par le Conseil de Recherche en Sciences Humaines du Canada.

1 Dans cet article, le masculien pluriel est utilisé de façon générique et sans intention de discrimination. 
principales se dégagent des analyses: le peu de valeur pronostique des variables sociodémographiques, la prépondérance marquée de la variable "rôle des caractéristiques du marché du travail dans l'atteinte des objectifs" pour prédire la qualité de l'insertion et, finalement, l'importance prédictive du lien de l'emploi avec la formation.

\section{ABSTRACT}

Since the issues related to socioprofessional integration extend well over objective facts of reality and are also influenced subjective facts of one's reality, it is important to identify those facts that allow prediction of the quality of the socioprofessional integration of new workers. The objective of this article is to present an exploratory schéma gathering three types of variables defining quality of socioprofessional integration and three other types of variables that are likely to predict this quality. This schéma was tested with a subsample of 97 new workers, all graduated from higher education programmes. Results from regression analyses have enlighted the relative strenght of some objective and subjective criteria and show that all the schéma's variables except age contribute to the understanding of socioprofessional integration of young graduated. Three major findings came out from the analyses: the low predictive value of demographic variables, the marked preponderance of the variable "role of job market's characteristics in the attainment of one's goals" in predicting professional integration quality and, finally, the predictive importance of the link between job and school training.

La transition de la formation initiale à la vie active est une étape cruciale dans le cheminement professionnel de l'individu, notamment parce que c'est à cette étape qu'il tente de mettre en application les compétences acquises au cours de sa formation initiale (Fournier \& Bellerive, 1991). Pour l'OCDE (1996), la transition de la formation initiale à la vie active réfère à la période durant laquelle les jeunes passent d'une activité principale, représentée par la scolarité à temps plein (ou son équivalent professionnel), à une autre activité principale, représentée par le travail. Pour plusieurs auteurs, l'insertion professionnelle n'est plus considérée comme un moment plus ou moins invariable et prévisible, sans discontinuité, au cours duquel s'effectue le passage du système éducatif au système productif. Elle apparaît plutôt comme un processus complexe qui se déroule sur une période où s'enchevêtrent des situations 
de recherche d'emploi, d'emploi, de chômage, de formation et d'inactivité (Trottier, Perron, \& Diambomba, 1995). De son côté, Vincens (1998) insiste sur le fait que la conception de l'insertion socioprofessionnelle ne peut se réduire uniquement à un processus de stabilisation sur le marché du travail mais doit également inclure l'idée de socialisation professionnelle en plus de prendre en compte la question de l'autonomie financière de même que la probabilité de la maintenir. La présente étude s'inscrit dans la foulée d'une recherche longitudinale menée par Fournier et Croteau (1993-2000) auprès de jeunes diplômés des secteurs secondaire professionnel, collégial technique et universitaire. L'analyse d'une première génération de résultats a permis d'observer que même en possession d'un emploi en lien avec la formation, $60 \%$ des diplômés se considèrent toujours en recherche d'emploi, suggérant alors que l'occupation d'un emploi ne peut, à elle seule, être garante d'une insertion socioprofessionnelle satisfaisante. D'une deuxième génération de résultats obtenus avec la même population, trois ans après l'obtention du diplôme, il ressort que plusieurs sujets utilisent de nombreux critères autres que ceux qui sont reliés à l'emploi (permanence, lien avec la formation etc.) pour définir une insertion socio-professionnelle stable et compléte. Ces critères se rattachent le plus souvent à l'atteinte d'une forme de qualité de vie tant personnelle (possibilités de réaliser ses projets, sentiment d'équilibre entre les différentes sphères de vie, sécurité et indépendance financière, etc.) que professionnelle (sentiment de réalisation au travail peu importe le statut de l'emploi, maîtrise des compétences dans son domaine, etc.). Ainsi, l'idée de l'insertion socioprofessionnelle comme d'un processus qui s'étend sur plusieurs années continue à s'imposer et, avec elle, l'idée qu'une insertion professionnelle compléte et réussie s'associe autant à un état subjectif individuel qu'à une situation objective, facilement observable.

En somme, si les enjeux liés à la satisfaction de l'insertion socioprofessionnelle s'étendent bien au delà de données factuelles (type d'emploi occupé, salaire, type d'entreprises, etc.) et sont aussi influencés par des données inscrites dans la réalité subjective de l'individu, il semble important d'identifier ces données qui permettent justement de prédire la qualité de l'insertion de ceux et celles qui sont au travail. Plus précisément, à une époque marquée par la précarité de l'emploi et par l'incertitude du marché du travail, il faut se demander quelles sont les variables qui permettent le mieux de prédire la qualité de l'insertion 
socioprofessionnelle des nouveaux travailleurs diplômés en précisant la force relative des critères objectifs comme le sexe du sujet et le statut de l'emploi et celle des critères plus subjectifs comme la perception des facteurs facilitants, dans la prédiction de la qualité de l'insertion, spécifiquement de ceux et celles qui sont sur le marché du travail. En effet, si un nombre relativement important d'études porte sur l'identification de facteurs prédictifs ou favorables à l'obtention d'un emploi à la sortie de la formation initiale, il existe, à notre connaissance, une rareté d'études portant spécifiquement sur la qualité de l'insertion de ceux et celles qui ont obtenu ces emplois. C'est ce à quoi s'intéresse le présent article qui fait état des résultats d'une première investigation d'un schéma exploratoire mettant à contribution tant des critères objectifs que subjectifs pour prédire la qualité de l'insertion de nouveaux travailleurs et de nouvelles travailleuses diplômés.

\section{CONTEXTE THÉORIQUE}

La sortie du système éducatif représente pour beaucoup de jeunes, une période difficile car, avec comme seul bagage le diplôme, ils sont souvent surpris et déçus, ayant tellement investi et nourri d'attentes à l'égard du travail et du marché du travail en comparaison de ce que le marché du travail peut réellement leur offrir (Fournier \& Bellerive, 1991). En effet, d'un côté et en dépit de la reconnaissance de la conjoncture économique difficile, plusieurs jeunes diplômés entretiennent des attentes élevées vis-à-vis du travail et du marché du travail, attentes qui dépassent largement l'obtention de gains financiers et s'associent généralement à des notions de réalisation et de développement personnels. D'un autre côté, il semble bien que l'emploi à temps partiel ou contractuel caractérise les premières expériences des jeunes à la sortie du système de formation et que cette situation ait tendance à se prolonger dans la vie des jeunes diplômés, les débouchés dans les secteurs qui leur étaient ouverts ces dernières décennies se faisant de plus en plus rares (Gauthier, 1992). Ainsi, dans le contexte difficile qui prévaut actuellement sur le marché du travail, le diplôme offre de moins en moins l'assurance d'un emploi régulier, lequel facilite notamment la mise en place de projets de vie. Curie (1993) souligne à ce sujet que la relation entre l'emploi et les compétences acquises dans le système de formation devient de plus en plus ténue et que l'accès à un emploi stable est trop souvent précédé par l'exercice de plusieurs 
emplois précaires et à temps partiel, obligeant le nouvel arrivant à accepter ce qu'on lui offre et le confinant dans une situation professionnelle instable, offrant très peu de possibilités d'action. Ajoutant à ce propos, Tremblay (1994) fait remarquer que l'âge moyen auquel les jeunes adultes québécois arrivent à trouver une forme de stabilité en emploi se situe aujourd'hui entre 25 et 30 ans alors qu'elle se situait autour de la vingtaine il n'y a pas si longtemps. Cette situation professionnelle instable et marquée par la discontinuité, affecte, particulièrement à cet âge où les projets sont à construire, la stabilité et l'emprise sur la situation personnelle.

Plusieurs variables sont susceptibles d'affecter la qualité de l'insertion socioprofessionnelle des nouveaux travailleurs diplômés. Trois groupes de variables indépendantes ont retenu l'attention dans le cadre de la présente étude pour tenter de prédire la qualité de l'insertion socioprofessionnelle de ces sujets. Le premier groupe concerne les variables sociodémographiques, soit le sexe, l'âge et le niveau de scolarité du sujet. Le second touche trois variables liées à l'emploi, à savoir le lien de l'emploi occupé avec la formation, le type d'emploi et le revenu. Enfin, le troisième groupe de variables renvoie à la perception des sujets quant au rôle facilitant de trois facteurs dans l'atteinte des objectifs personnels et professionnels. Ces facteurs sont l'environnement social du sujet, certains attributs personnels et certaines caractéristiques du marché du travail.

Concernant les variables sociodémographiques, les résultats de plusieurs études indiquent que les femmes sont en moins bonne posture d'insertion puisque, comme le fait remarquer Bengle (1991), à compétences égales, elles ont de moins bons emplois que les hommes et, lorsqu'elles occupent des emplois comparables, elles sont moins bien rémunérées. Les hommes ont aussi encore plus de chances de se trouver un emploi permanent et à temps plein que les femmes (Bengle, 1991; Tanguay, 1986). Ces différences tendent à s'accentuer lorsque le niveau de scolarisation augmente (Trottier et al., 1995). Enfin, Tremblay (1994) rapporte que malgré leur plus faible représentation sur le marché du travail, les femmes comptent pour plus de la moitié (54\%) des personnes ayant des formes d'emploi atypiques au Canada et au Québec.

Relativement à l'âge des sujets, même si les enquêtes récentes montrent que les jeunes de 15 à 24 ans représentent $40 \%$ des personnes ayant des formes d'emploi atypiques, il existe actuellement une rareté 
évidente de recherches portant sur les effets de l'âge sur la satisfaction au travail dans les premières années de vie professionnelle. Cette rareté de recherches a aussi été observée par Touzard, Lancry-Hoestlandt, Depolo et Sarchielli (1996). Dans le cadre d'une étude internationale portant sur la socialisation au travail de jeunes dans leur premier emploi, ces chercheurs ont trouvé, contrairement à l'hypothèse qu'ils avaient formulée, que les jeunes travailleurs et travailleures débutants (16-19 ans) sont plus satisfaits, mieux dans leur peau et planifient davantage leur carrière que les travailleurs et travailleures débutants plus âgés (22 ans et plus). De plus, ces auteurs ont observé que c'est plutôt l'âge conjugué au changement dans le travail qui affectent les variables de satisfaction. Ainsi, plus le travailleur ou la travailleuse est jeune, plus il ou elle se montre satisfait-e et le changement de travail ou d'emploi ajoute au niveau de satisfaction. De plus, et toujours à l'inverse de ce qu'ils avaient postulé, ce sont les plus jeunes qui s'adaptent le mieux aux changements dans les premières années de travail. Toutefois, comme l'a fait remarquer Bengle (1991) il y a quelques années, les rares recherches concernant les effets de l'âge sur l'insertion sont à elles seules peu concluantes car l'âge en lui-même n'a d'effets réels qu'à travers les multiples variables qui lui sont liées. Cependant, cet auteur fait tout de même remarquer que le niveau de rémunération permet de distinguer les travailleurs et les travailleuses plus jeunes de leurs collègues plus âgés, les premiers ayant généralement un revenu moindre que les seconds. En somme, la relation entre l'âge et l'expérience de l'insertion socioprofessionnelle est plus complexe qu'elle n'apparaît à première vue et mérite d'être examinée.

Enfin, en ce qui a trait à la scolarité, la grande majorité des études consultées fait ressortir que la situation des non diplômés est toujours plus précaire que celle des diplômés. De fait, plus les jeunes sont scolarisés, plus leur participation au marché du travail augmente (Cairns, Woodward, \& Hashizume, 1992), la scolarité demeurant un moyen privilégié d'alléger leurs difficultés sur le marché de l'emploi (Bengle, 1991; Trottier et al., 1995). Ajoutant à ce propos, un rapport récent de l'OCDE (1996) fait remarquer que les taux de chômage dans les sociétés industrialisées varient non seulement selon le programme d'études choisi mais aussi selon le niveau de formation acquise, les plus scolarisés étant moins touchés par le chômage. Toutefois, les chercheurs sont de plus en plus nombreux à décrier le manque de fiabilité du taux de chômage 
relativement faible des nouveaux diplômés pour rendre compte de leur insertion socioprofessionnelle, ces derniers étant souvent obligés d'accepter des emplois peu en lien ou en très en deçà du niveau de leur formation (Fournier \& Croteau, 1997; OCDE, 1996; Trottier et al., 1995). En somme, la relation entre l'emploi et le niveau de scolarité est complexe. Si le diplôme facilite l'obtention d'un emploi à la sortie du système éducatif, il appert que ces emplois sont souvent en-dessous du niveau de formation et des attentes.

Le contexte économique se caractérisant actuellement par une fragilisation des emplois, les emplois créés étant le plus souvent à temps partiel et à faible revenu, trois variables liées à l'emploi occupé ont paru particulièrement susceptibles d'affecter la qualité de l'insertion des nouveaux travailleurs. D'abord, le lien de l'emploi avec la formation. En effet, plusieurs auteurs font observer que les diplômés sont souvent obligés d'accepter des emplois qui n'ont rien à voir avec leur formation (Galland, 1995; OCDE, 1996; Trottier et al. 1995), affectant du même souffle leurs projets personnels et professionnels. De plus, de nombreuses enquêtes mettent en évidence l'augmentation du nombre de jeunes qui occupent des emplois à temps partiel depuis les 10 dernières années (Conseil permanent de la jeunesse, 1997; Matte, Baldino \& Courchesne, 1998; Moreau, 1997), ce qui contribue à appauvrir les travailleurs et travailleuses et à les garder dans un état d'insécurité financière, les rendant du même coup plus vulnérables sur le plan personnel. Découlant logiquement de la variable précédente, le revenu annuel brut apparaît comme une variable susceptible d'affecter la qualité de l'insertion socioprofessionnelle des travailleurs et travailleuses, principalement par le biais du sentiment de contrôle de leur situation financière et de la possibilité d'élaborer des projets personnels.

Le troisième groupe de variables, finalement, reflète les perceptions des sujets quant au rôle de l'environnement social, de certains attributs personnels et de différentes caractéristiques du marché du travail dans l'atteinte de leurs objectifs personnels et professionnels. Concernant le rôle de l'environnement social, des entrevues effectuées par Gauthier (1994) auprès de jeunes chômeurs et de jeunes qui ont réussi leur insertion en emploi, suggèrent que la famille est particulièrement importante dans cette période de transition et que l'absence de soutien peut rendre l'insertion socioprofessionnelle plus difficile à différents égards. En effet, il semble que le fait de vivre seul, sans soutien, fait 
accepter par nécessité des emplois dont on n'aurait pas voulu en d'autres circonstances et peut expliquer, en partie du moins, le sentiment d'échec et la déception ressentie vis-à-vis de l'emploi occupé. Il semble aussi que les pressions exercées par l'entourage pour inciter le jeune à dénicher un emploi et à quérir son indépendance soient associées à un moins grand bien-être psychologique (Cairns et al., 1992). En outre, les auteurs s'entendent généralement pour reconnaître le lien étroit existant entre la présence de soutien social et le bien-être de l'individu (Coyne \& Bolger, 1990; Coyne \& De Longis, 1986; Gauthier, 1994). Finalement, il semble que le soutien social, particulièrement le soutien social perçu, a un impact sur le bien-être psychologique de l'individu (Pierce, Sarason, \& Sarason, 1991; Sarason, Pierce, \& Sarason, 1991), possède un effet tampon sur le stress associé à l'insertion (Rascle, 1994) et influence l'intensité de même que le choix des moyens mis en oeuvre lors de la recherche d'emploi (Gauthier, 1992).

Le rôle joué par la perception de certains attributs individuels dans l'atteinte des objectifs personnels et professionnels est également à considérer à titre de variable pouvant influencer la qualité de l'insertion socioprofessionnelle. À ce sujet, certaines variables perceptuelles peuvent être liées à la satisfaction et au bien-être psychologique que retire l'individu de son travail (Bruhn, 1989). Selon cet auteur, le sentiment d'apprendre et de s'actualiser et la perception de contribuer à quelque chose ayant un sens, de réussir et de s'accomplir, participent au développement et au maintien du sentiment de mieux-être chez l'individu. Dans la même veine, Kabanoff (1982) souligne que la valeur d'un travail repose d'abord sur la possibilité que perçoit une personne d'utiliser ses habiletés, ses connaissances et ses capacités. Finalement, le rôle perçu de certaines caractéristiques du marché du travail dans l'atteinte des objectifs personnels et professionnels s'ajoute comme variable pouvant influencer la qualité de l'insertion socioprofessionnelle. À cet égard, Fournier et Croteau (1997) ont constaté que la perception des jeunes en regard du marché du travail est une des variables les plus reliées à l'obtention d'un emploi en lien avec leur formation. Cette étude, tout comme les autres mentionnées précédemment, sont parmi les rares à s'être intéressées à l'impact des perceptions reliées au marché du travail sur la manière dont est vécue l'insertion socioprofessionnelle. Dans la mesure où le marché du travail se présente comme particulièrement contraignant pour les jeunes, il est intéressant 
d'explorer si justement le fait de percevoir certaines caractéristiques du marché du travail (ex: conditions d'emploi proposées) comme facteur facilitant ou comme obstacle, influence la satisfaction professionnelle, la satisfaction personnelle et le sentiment de contrôle de la situation financière des nouveaux travailleurs et des nouvelles travailleuses.

Trois groupes de variables dépendantes ont servi à définir la qualité de l'insertion socioprofessionnelle des nouveaux travailleurs et les nouvelles travailleuses diplômés. Le premier groupe de variables concerne la satisfaction professionnelle, le second réfère à la satisfaction personnelle retirée de la situation d'emploi et le troisième renvoie au sentiment de contrôle face à la situation financière que permet la situation professionnelle.

En ce qui a trait à la satisfaction professionnelle, l'étude de Touzard et al. (1996), notamment, fait écho à nos préoccupations. En effet, cette équipe de chercheurs a tenté de clarifier la dynamique de la satisfaction au travail des jeunes débutant leur vie professionnelle et de mieux comprendre les effets de différents types de variables sur leur insertion en emploi. Tout comme il est proposé de le faire ici, ces auteurs ont principalement eu recours aux perceptions des jeunes en regard de leur emploi et de leur environnement immédiat de travail pour définir la dynamique de la satisfaction au travail. Plus précisément, dans la présente étude, la notion de satisfaction professionnelle s'exprime par le degré de satisfaction des jeunes travailleurs et travailleuses face aux éléments intrinsèques et extrinsèques de leur emploi, par les écarts entre les attentes et la situation réelle d'emploi, par leur désir ou non de conserver leur emploi, de même que par le sentiment général d'atteinte ou non de leurs objectifs personnels et professionnels et ce, trois ans après leur sortie du système éducatif. La deuxième variable dépendante, soit la satisfaction personnelle retirée de la situation d'emploi, est d'abord cernée par un indicateur lié à l'influence de l'emploi sur différentes facettes associées au bien-être psychologique de l'individu. Ensuite, prenant notamment appui sur les travaux de Hartnagel et Krahn (1995), la satisfaction personnelle retirée de la situation d'emploi est mesurée par un indice traduisant jusqu'à quel point la situation d'emploi affecte les possibilités de réaliser des projets personnels. En effet, comme le rapportent ces auteurs, le fait d'occuper un emploi rémunéré s'avère déterminant pour acquérir l'indépendance face à la famille et pour jouer les différents rôles sociaux qu'appelle le passage à l'âge adulte (mariage 
ou vie commune avec un conjoint, parentalité, etc.). La satisfaction personnelle sera donc évaluée à partir d'indices liés au bien-être psychologique procuré par l'emploi et par l'influence perçue de la situation d'emploi sur la possibilité de réaliser des projets personnels.

La troisième variable dépendante, finalement, reflète le sentiment de contrôle face à la situation financière et est apparue en lien étroit avec la qualité de l'insertion socioprofessionnelle. En effet, bien que bon nombre d'études aient clairement mis en évidence l'existence d'une relation entre l'âge, le niveau de formation et le revenu (Vincens, 1996), il semble bien que cette relation n'ait plus la même importance aujourd'hui puisque la précarité des emplois ne permet plus, bien souvent, d'assurer un revenu continu et permanent. De plus, le taux d'endettement général particulièrement élevé des étudiantes et des étudiants de niveau collégial et de l'université (Conseil Permanent de la Jeunesse, 1997) oblige à relativiser la relation formation-emploi-revenu, un revenu d'emploi élevé n'apportant pas toujours la sécurité financière escomptée. Dans cette optique, il devient important de s'attarder non plus uniquement au revenu d'emploi mais aussi à la perception qu'a la personne de sa situation financière pour définir et comprendre toutes les nuances liées à la qualité de son insertion socioprofessionnelle. Le sentiment de contrôle face à la situation financière sera mesuré par les indicateurs suivants: satisfaction face à la situation financière, perception de l'équilibre financier et précarité perçue.

Les relations discutées ci-haut relativement à la qualité de l'insertion socioprofessionnelle peuvent être schématisées tel que le présente la figure 1.

\section{MÉTHODOLOGIE}

\section{Formation de l'échantillon}

La première année de la recherche, les sujets ont été invités à participer à la recherche sur une base volontaire. Tous les sujets sélectionnés répondent aux critères suivants: 1) être inscrit dans un programme d'études menant directement à l'exercice d'un métier ou d'une profession (secondaire professionnel, collégial technique, baccalauréat spécialisé), 2) être à la dernière année d'études prévue avant l'obtention du diplôme convoité et, 3) être inscrit à temps plein dans le programme d'études. En tout, 205 sujets provenant de 21 
Figure 1

Schéma exploratoire de la qualité de l'insertion de nouveaux travailleurs diplômés

\section{VARIABLES INDÉPENDANTES}
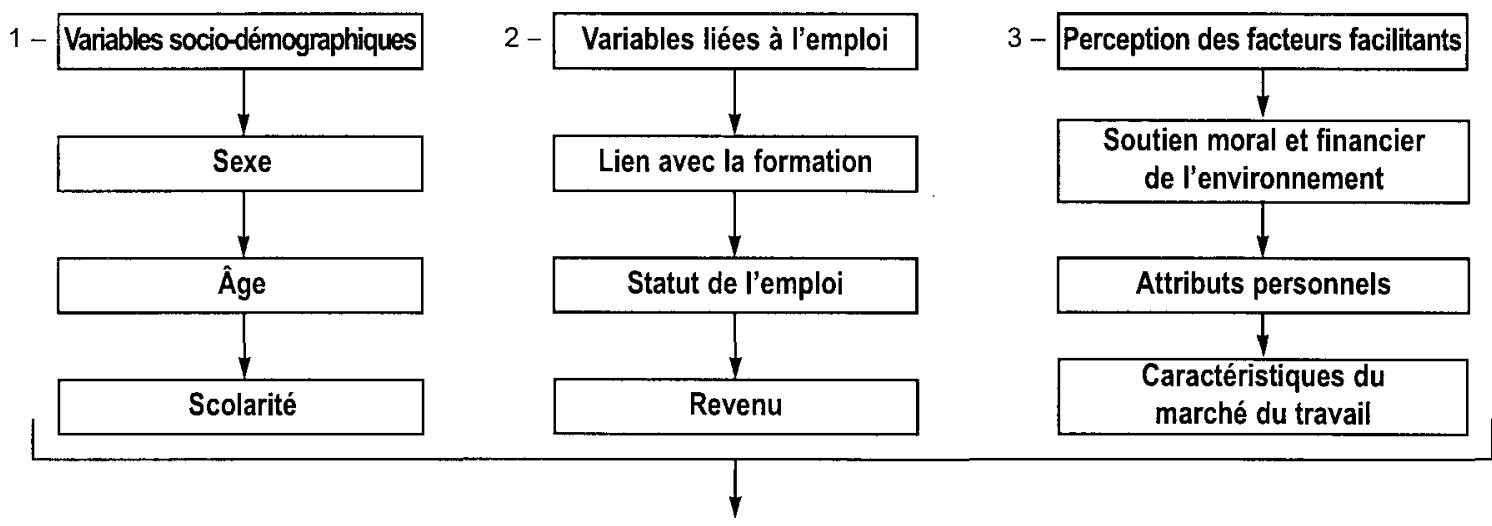

VARIABLES DÉPENDANTES
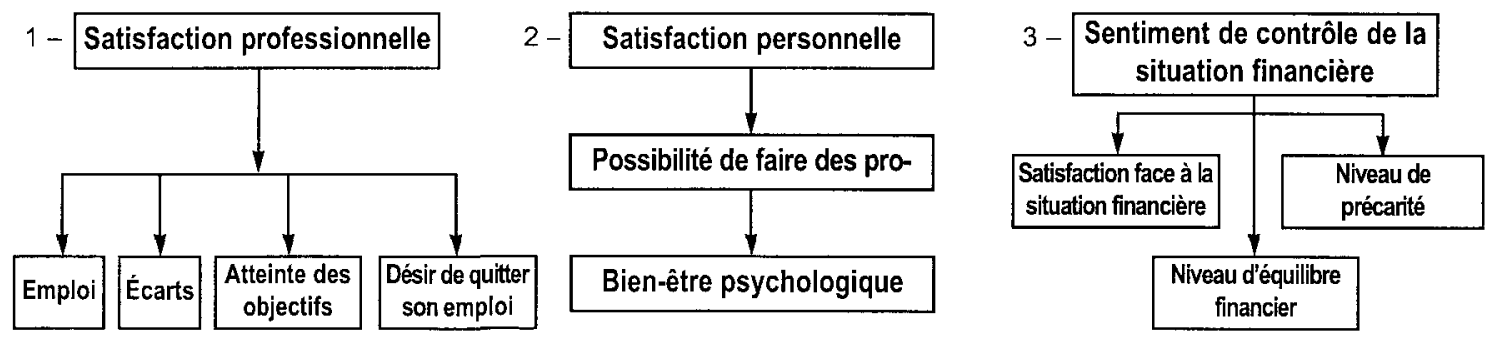
programmes d'études différents ont été recrutés lors de cette première année de recherche et ont constitué l'échantillon initial. La troisième année de la recherche, tous les sujets qui forment l'échantillon initial ont été rejoints par téléphone pour la deuxième série d'entrevues. En tout, 190 sujets ont accepté de maintenir leur participation à la recherche et constituent donc l'échantillon de la troisième année. La cinquième année de la recherche, la même procédure a été utilisée (entrevues et questionnaires). En tout, 152 sujets ont accepté l'invitation de poursuivre leur engagement dans la recherche et ont été interviewés. Cependant, seulement 134 des sujets interviewés ont retourné les questionnaires. Les résultats présentés ici sont tirés de données recueillies lors de la cinquième année de l'étude, trois ans après la diplômation des sujets et concernent exclusivement les 97 d'entre eux qui sont en emploi, soit $72,6 \%$ de l'échantillon qui a retourné les questionnaires.

Parmi ces 97 sujets, $74 \%$ sont des femmes, un peu moins du tiers $(27.8 \%)$ a terminé une formation secondaire professionnelle, un peu plus du tiers $(37,1 \%)$ a terminé une formation collégiale technique et, finalement, un tiers $(35,1 \%)$ a obtenu un diplôme universitaire. Plus de la moitié des sujets (58.8\%) est âgée de 22 à 25 ans, les autres étant en très grande majorité, légèrement plus âgés.

\section{DESCRIPTION DES VARIABLES}

\section{Variables indépendantes}

1. Variables socio-démographiques. Il s'agit du sexe des sujets (féminin, masculin), de leur âge ( 25 ans et moins, plus de 25 ans) et de leur niveau de scolarité (secondaire, collégial, universitaire).

2. Variables liées à l'emploi. Ces variables incluent le degré de relation perçu entre cet emploi et la formation respective des sujets, sur une échelle en quatre points (de "totalement en lien" à "pas du tout en lien"), le type d'emploi (temps plein, temps partiel) et le revenu annuel brut gagné en dollars canadiens.

3. Perception des facteurs facilitants. Cette catégorie, d'ordre plus psychologique, repose sur trois variables, toutes perceptuelles, lesquelles décrivent des facteurs pouvant éventuellement faciliter l'atteinte des objectifs du répondant ou de la répondante: a) le soutien moral et financier de l'environnement familial, b) certains attributs personnels de l'individu, et finalement, c) certaines caractéristiques du marché du 
travail. Chacun de ces trois facteurs présentait un certain nombre d'énoncés visant à le détailler. Chaque énoncé était associé à une échelle de réponses en quatre points, allant de "très négativement" à "très positivement".

\section{Variables dépendantes}

Les variables dépendantes sont regroupées en trois blocs permettant de qualifier la qualité de l'insertion socioprofessionnelle, soient: 1) la satisfaction professionnelle, 2) la satisfaction personnelle et 3) le contrôle de la situation financière.

\section{Satisfaction professionnelle}

Ce bloc propose quatre variables: 1) la satisfaction reliée à l'emploi, 2) l'écart perçu entre les attentes et la réalité, 3) le sentiment d'avoir ou non atteint ses objectifs, et 4) l'intention de quitter son emploi.

1. Satisfaction reliée à l'emploi. Suite à la consigne: Jusqu'à quel point vous sentez-vous satisfait-e de. . . les sujets devaient évaluer, sur une échelle en quatre points (de "très satisfait" à "insatisfait"), leur degré de satisfaction en regard de chacun de 4 facteurs intrinsèques (ex.: l'utilisation du potentiel) et de 5 facteurs extrinsèques (ex.: rapport avec les supérieurs, chances d'avancement). Les indices de consistance interne obtenus atteignent $\alpha=0.85$ (fac. intrinsèques) et $\alpha=0.65$ (fac. extrinsèques).

2. Écart perçu entre les attentes et la réalité. Présentant 19 items dont 11 facteurs intrinsèques (ex.: niveau d'autonomie professionnelle, sentiment de confiance en soi) et 8 facteurs extrinsèques (ex.: style de gestion de l'entreprise, salaire), cette échelle était accompagnée de la consigne suivante: En comparant votre situation actuelle à ce que vous espériez avant d'être sur le marché du travail, dans l'ensemble, quelle évaluation en faites-vous. Les réponses étaient exprimées sur une échelle en quatre points (de "nettement pire que j'espérais" à "nettement mieux que j'espérais"). Le coefficient alpha obtenu est de $\alpha=0.90$ pour les facteurs intrinsèques et de $\alpha=0.78$ pour les facteurs extrinsèques.

3. Sentiment d'avoir ou non atteint ses objectifs. Cette variable est mesurée par la question: De façon générale, avez-vous l'impression de rencontrer vos objectifs personnels et professionnels? L'échelle de réponse est en quatre points (de "pas du tout" à "beaucoup"). 
4. Intention de quitter son emploi. Cette dernière question du bloc relié à la satisfaction professionnelle est mesurée par la question suivante: Désirez-vous quitter votre emploi? Le choix de réponse est dichotomique (oui/non).

\section{Satisfaction personnelle}

Ce bloc compte deux variables: 1) la possibilité de faire des projets et 2) le bien-être personnel.

1. Possibilité de faire des projets. Cette variable est mesurée par cinq items, évalués sur une échelle en quatre points (de "très négativement" à "très positivement") à laquelle s'adjoint l'option "Ne s'applique pas". Les sujets devaient répondre à la question: De quelle façon votre situation d'emploi actuelle affecte-t-elle vos projets. Les cinq projets énumérés ont été choisis en regard de leur pertinence dans la vie de jeunes adultes de 18-30 ans (ex.: devenir autonome par rapport à son milieu familial, s'établir avec un-e conjoint-e). Le coefficient alpha obtenu pour ces cinq items est de $\alpha=0.87$.

2. Bien-être psychologique. Cette variable est mesurée par cinq items associés à la question: De quelle façon, votre situation d'emploi actuelle affecte-t-elle votre. . . Les cinq items énumérés (ex.: sentiment de sécurité personnelle, niveau de stress) sont évalués sur une échelle en cinq points (de "très négativement" à "très positivement"). Le coefficient alpha obtenu pour ces cinq items est de $\alpha=0.81$.

\section{Sentiment de contrôle de la situation financière}

Ce dernier bloc regroupe trois variables: 1) la satisfaction générale face à la situation financière, 2) le niveau d' équilibre financier, et 3) le niveau de précarité perçu.

1. Satisfaction générale face à la situation financière. Cet indicateur est associé à l'énoncé Comment vous sentez-vous par rapport à votre situation financière? et à une échelle de réponse en quatre points ( "Je me sens privilégié-e par ma situation financière actuelle" à "je me sens découragé-e face à ....”).

2. Niveau d'équilibre financier. Cet indicateur est évalué par l'énoncé Votre situation financière se caractérise présentement par. . . auquel est associé une échelle de réponse en six points (de "obligations financières beaucoup trop élevées compte tenu des revenus" à "obligations financières très en dessous des revenus" avec une option "pas d'obligations financières"). 
3. Niveau de précarité perçu. Cet indicateur est mesuré par l'énoncé "Jusqu'à quel point considérez-vous votre situation financière comme précaire?" et est associé à une échelle de réponse en quatre points (de "pas du tout" à "totalement").

\section{RÉSULTATS}

La présentation des résultats se divise en deux parties. D'abord, les données descriptives permettent de dégager la tendance générale des répondants et des répondantes par rapportaux différentes variables à l'étude. En second lieu, les analyses de régression multiple permettent de vérifier la capacité pronostique respective des variables sociodémographiques, des variables liées à l'emploi et des variables perceptuelles associées aux facteurs facilitants sur les variables liées à la qualité de l'insertion socioprofessionnelle.

Le tableau 1 présente les données descriptives obtenues, pour chacune des variables dépendantes à l'étude, pour l'échantillon global de même qu'en fonction du sexe et du niveau de scolarité des répondants et répondantes. Pour chacune des variables également, le test $\mathrm{F}$ et le test $t$ indiquent les différences existantes selon le niveau de scolarité et le sexe des répondants et répondantes.

Pour l'ensemble des indices de satisfaction, les moyennes obtenues par les sujets permettent de présenter une image relativement positive de la qualité de l'insertion des jeunes diplômés. Ainsi, les différentes variables reliées à la satisfaction professionnelle indiquent que la majorité des sujets se sentent satisfaits des aspects intrinsèques et extrinsèques de l'emploi occupé, que ce qu'ils expérimentent à leur emploi est généralement mieux qu'ils ne l'espéraient et, finalement, que très peu d'entre eux expriment l'intention de quitter leur emploi. Les résultats à la variable "sentiment d'atteindre ou non ses objectifs" laissent entrevoir un portrait moins positif, par contre. En effet, la majorité des sujets estiment que leur situation professionnelle actuelle, toute satisfaisante qu'elle semble être, ne leur permet que "moyennement" d'atteindre les objectifs qu'ils s'étaient fixés alors qu'ils étaient aux études. La dispersion élevée des résultats de cette variable en particulier, en outre, suggère que les répondants ne partagent pas tous cette opinion et que, du fait, plusieurs $(29,5 \%)$ ont plutôt précisé que leur situation actuelle ne permettait "qu'un peu" l'atteinte de leurs objectifs. 
Tableau 1

Moyenne, écart-type et tests de différences des moyennes des variables liées à la qualité de l'insertion socioprofessionnelle selon le niveau de scolarité et le sexe des sujets

\begin{tabular}{|c|c|c|c|c|c|c|c|c|c|c|c|c|c|c|}
\hline \multirow[b]{3}{*}{ Variables } & \multicolumn{6}{|c|}{ Niveau de scolarité } & \multirow{2}{*}{\multicolumn{2}{|c|}{ Universitaire }} & \multirow[t]{3}{*}{$\underline{F}$} & \multicolumn{4}{|c|}{$\underline{\text { Sexe }}$} & \multirow[t]{3}{*}{$\underline{1}$} \\
\hline & \multicolumn{2}{|c|}{ Global } & \multicolumn{2}{|c|}{ Secondaire } & \multicolumn{2}{|c|}{ Collégial } & & & & \multicolumn{2}{|c|}{ Hommes } & \multicolumn{2}{|c|}{ Femmes } & \\
\hline & $\mathrm{m}$. & é.-t. & $\mathrm{m}$. & é.t. & $\mathrm{m}$. & é.-t. & $\mathrm{m}$. & é.-t. & & m. & é.-t. & & é.-t. & \\
\hline \multicolumn{15}{|l|}{ SATISFACTION PROFESSIONNELLE } \\
\hline 1. Satisfaction intrinsèque de l'emploi (max.: 4) & 3,0 & 0,77 & $\underline{3.2}$ & 0,71 & $\underline{2,8}$ & 0,84 & 2,9 & 0,68 & $3,2 *$ & 3,0 & 0,74 & 3,0 & 0,78 & $-0,0$ \\
\hline Satisfaction extrinsèque de l'emploi (max.: 4) & 3,1 & 0,45 & $\overrightarrow{3,2}$ & 0,40 & $\overline{3,0}$ & 0,47 & 1,9 & 0,43 & 2,6 & 3,2 & 0,44 & 3,0 & 0,45 & $-1,8$ \\
\hline 2. Écarts entre attentes et réalité (intrins) (max.: 4) & 3,0 & 0,49 & 3.2 & 0,43 & $\underline{2.8}$ & 0,52 & 3,1 & 0,44 & $5,1 *$ & 3,0 & 0,51 & 3,0 & 0,49 & 0,3 \\
\hline Écarts entre attentes et réalité (extrin) (max.: 4) & 2,8 & 0,43 & $\overline{3,0}$ & 0,45 & $\overline{2,7}$ & 0,43 & 2,9 & 0,39 & 2,5 & 2,9 & 1,00 & 2,8 & 0,43 & 1,0 \\
\hline 3. Sentiment d'atteinte ou non ses objectifs (max.: 4) & 2,9 & 0,95 & 3,0 & 1,00 & 2,7 & 1,01 & 3,2 & 0,78 & 2,2 & 2,9 & 1,00 & 2,9 & 0,95 & 0,2 \\
\hline 4. Intention de quitter son emploi (max.:2) & 1,7 & 0,46 & 1,8 & 0,41 & 1,6 & 0,50 & 1,8 & 0,43 & 1,6 & 1,8 & 0,42 & 1,7 & 0,47 & 1,0 \\
\hline \multicolumn{15}{|l|}{ SATISFACTION PERSONNELLE } \\
\hline 5. Impact sur les projets de vie (max:: 4) & 3,0 & 0,82 & 3,2 & 0,68 & 2,9 & 0,85 & 2,9 & 0,88 & 0,9 & 3,1 & 0,79 & 2,9 & 0,83 & 0,9 \\
\hline Quitter domicile familial $(n=34)$ & 3,1 & 1,04 & 3,3 & 0,98 & 2,8 & 1,11 & 3,2 & 1,03 & 0,7 & 2,9 & 0,99 & 3,2 & 1,06 & $-0,8$ \\
\hline Débuter vie commune avec conjoint-e $(n=41)$ & 3,1 & 1,00 & 3,5 & 0,93 & 2,7 & 1,10 & 3,5 & 0,69 & 2,8 & 3,2 & 0,83 & 3,1 & 1,06 & 0,3 \\
\hline Acheter des biens coûteux ( $\mathrm{n}=89$ ) & 3,0 & 0,95 & 3,3 & 0,74 & 3,0 & 0,95 & 2,7 & 1,07 & 2,4 & 3,2 & 0,75 & 2,9 & 1,00 & $-1,4$ \\
\hline Effectuer des activités de loisirs $(n=93)$ & 3,0 & 0,81 & 3,0 & 0,79 & 3,1 & 0,75 & 2,8 & 0,90 & 0,9 & 3,0 & 0,81 & 2,9 & 0,82 & 0,5 \\
\hline Avoir des enfants $(n=56)$ & 2,8 & 1,00 & 2,9 & 1,05 & 2,6 & 1,05 & 2,9 & 0,94 & 0,7 & 2,8 & 1,17 & 2,8 & 0,97 & $-0,1$ \\
\hline 6. Impact sur le bien-être psychologique (max.: 5) & 3,6 & 0,77 & 3,8 & 0,76 & 3,3 & 0,83 & 3,7 & 0,58 & 4,3 & 3,7 & 0,63 & 3,5 & 0,80 & 0,9 \\
\hline Sentiment de sécurité personnelle & 3,7 & 1,02 & 3,8 & 0,98 & 3,4 & 1,11 & 3,8 & 0,92 & 1,5 & 3,8 & 0,93 & 3,6 & 1,06 & 0,5 \\
\hline Sentiment de bien-être personnel & 3,8 & 0,94 & 4,0 & 0,87 & 3,5 & 1,06 & 3,9 & 0,82 & 2,3 & 3,9 & 0,83 & 3,7 & 0,98 & 0,8 \\
\hline Sentiment d'autonomie personnelle & 3,9 & 0,98 & 4.2 & 0,86 & $\underline{3.6}$ & 1,08 & 4,1 & 0,82 & $4,3 *$ & 4,1 & 0,78 & 3,9 & 1,03 & 1,1 \\
\hline Sentiment de réalisation personnelle & 3,7 & 1,05 & $\overline{4.1}$ & 0,71 & $\overline{3.3}$ & 1,17 & 3,9 & 1,01 & $5,2 *$ & 3,9 & 0,86 & 3,7 & 1,11 & 1,1 \\
\hline Niveau de stress & 2,8 & 0,97 & 2,9 & 1,08 & 2,7 & 0,93 & 2,8 & 0,94 & 0,5 & 2,8 & 0,87 & 2,8 & 1,01 & 0,2 \\
\hline \multicolumn{3}{|c|}{ SENTIMENT DE CONTRÔLE DE LA SITUATION FINANCIERE } & & & & & & & & & & & & \\
\hline 7. Sentiment face à la sit, financière (max.: 4) & 2,8 & 0,75 & 2,7 & 0,82 & 2,6 & 0,76 & 2,9 & 0,65 & 1,6 & 2,8 & 0,65 & 2,8 & 0,78 & $-0,3$ \\
\hline 8. Caractéristique de la sit, financière (max.: 6) & 3,0 & 0,86 & 2,9 & 0,78 & 2,9 & 0,87 & 3,1 & 0,92 & 0,3 & 3,2 & 0,80 & 2,9 & 0,87 & 1,3 \\
\hline 9. Niveau de précarité ressenti (max.: 4) & 3,0 & 0,70 & 3,1 & 0,62 & $\underline{2.8}$ & 0,74 & 3,3 & 0,67 & $3,5^{* *}$ & 3,3 & 0,63 & 3,0 & 0,70 & $-2,3 *$ \\
\hline
\end{tabular}


Dans l'ensemble, les moyennes obtenues pour les variables liées à la satisfaction personnelle présentent également un portrait assez positif, suggérant que la situation d'emploi des jeunes travailleurs et travailleuses affecte plutôt positivement leur situation de vie personnelle. Cependant, à la différence des résultats obtenus pour la satisfaction professionnelle, la dispersion pour l'ensemble des variables de cette catégorie est élevée. Ce résultat indique qu'un certain nombre de jeunes travailleurs et travailleuses estime retirer moins, dans leur vie personnelle, de leur situation de travail et ce, spécifiquement en regard de la possibilité de réaliser des projets de vie et du sentiment de bien-être psychologique. Concrètement, par exemple, notons que $28,4 \%$ des sujets obtiennent, à la variable reflétant l'impact de l'emploi sur la réalisation des projets de vie, une moyenne inférieure à 2,5 , point qui représente la valeur neutre (impact ni positif ni négatif) de l'échelle.

Les moyennes obtenues pour les variables liées à la satisfaction face à la situation financière indiquent que si la majorité des jeunes travailleurs et travailleuses participant à cette étude semble satisfaite et en contrôle de sa situation financière, la dispersion moyennement élevée des résultats aux variables "sentiment face à la situation financière", "caractéristiques de la situation financière" et "niveau de précarité ressenti", suggère que la précarité et l'inquiétude demeurent présentes pour une partie de l'échantillon. Ainsi, par exemple, à la variable "satisfaction générale face à la situation financière", $32 \%$ des sujets se sont dit inquiets ou découragés de leur situation financière.

Finalement, notons que si le niveau de scolarité et le sexe des répondants et des répondantes n'ont, somme toute, que peu d'effet sur la qualité de l'insertion des jeunes travailleurs et travailleuses, il n'empêche que les femmes mentionnent en plus grand nombre qu'elles se sentent dans une situation financière précaire et que les quelques différences enregistrées en regard du niveau de scolarité sont toujours le lot des étudiants et des étudiantes de niveau collégial. Sur ce dernier point, il semble en effet que l'expérience d'emploi vécue par les jeunes travailleurs du secondaire soit davantage comparable à celle vécue par les répondants universitaires.

Les prochains tableaux présentent les résultats des régressions multiples effectuées sur les différentes variables dépendantes servant à mesurer la qualité de l'insertion socioprofessionnelle, tel que proposé dans le schéma de la présente étude. 


\section{Satisfaction professionnelle}

Les régressions multiples effectuées à partir des variables indépendantes nous permettent de constater que certaines d'entre elles prédisent significativement le niveau de satisfaction professionnelle lié aux facteurs intrinsèques ou extrinsèques de l'emploi, aux écarts perçus entre les attentes et la réalité en regard de l'emploi occupé de même qu'au sentiment général d'atteinte ou non des objectifs personnels et professionnels. Le tableau 2 présente les résultats des régressions effectuées séparément sur l'ensemble des variables liées à la satisfaction professionnelle.

Les résultats obtenus permettent de constater que, de façon générale, les variables indépendantes atteignent un niveau de prédiction de variance très satisfaisant. En effet, elles expliquent, en moyenne, $30 \%$ de la variance associée aux cinq indicateurs de la satisfaction professionnelle. En outre, ce taux de variance expliquée atteint même $40 \%$ pour la satisfaction liée aux facteurs intrinsèques de l'emploi.

L'effet des variables sociodémographiques sur les cinq indicateurs de la satisfaction professionnelle s'avère plutôt modeste. En ce qui concerne la variable "sexe", elle s'avère un prédicteur significatif de la satisfaction liée aux facteurs extrinsèques de l'emploi (ex.: conditions de travail). Ainsi, les femmes se disent plus satisfaites que les hommes des caractéristiques extrinsèques de leur emploi. Cette variable n'agit comme prédicteur sur aucune autre variable dépendante. De la même façon, le niveau de scolarité s'avère un prédicteur significatif uniquement pour un indicateur, soit la satisfaction liée aux facteurs intrinsèques de l'emploi (ex: utilisation du potentiel). Ainsi, la satisfaction est d'autant plus forte que les répondants et les répondantes ont complété un niveau de scolarité élevé. Finalement, l'âge des sujets ne contribue pas à la prédiction des différentes variables liées à la satisfaction professionnelle.

En ce qui concerne les variables liées à l'emploi, on note un effet de prédiction plus important que pour le groupe de variables précédent, bien que cet effet soit principalement restreint à l'une des variables indépendantes, le lien de l'emploi avec la formation (de 3,0 à 44,9\% de variance expliquée). Ainsi, cette variable prédit-elle quatre des cinq indicateurs de la satisfaction professionnelle tout en se classant toujours parmi les deux prédicteurs expliquant le plus haut taux de variance. Plus spécifiquement, plus le lien entre l'emploi et la formation s'accroît, 
Tableau 2

Régressions multiples linéaires des variables liées à la satisfaction professionnelle

\begin{tabular}{|c|c|c|c|c|c|c|c|c|c|c|}
\hline & \multirow[b]{3}{*}{ Prédicteur } & \multicolumn{3}{|c|}{ Satisfaction } & \multicolumn{4}{|c|}{$\underline{\text { Écart des attentes }}$} & \multirow{2}{*}{\multicolumn{2}{|c|}{$\begin{array}{l}\text { Atteinte des } \\
\text { objectifs }\end{array}$}} \\
\hline & & $\begin{array}{c}\text { Facteurs } \\
\text { intrinsèques }\end{array}$ & \multicolumn{2}{|c|}{$\begin{array}{c}\text { Facteurs } \\
\text { extrinsèques }\end{array}$} & \multicolumn{2}{|c|}{$\begin{array}{c}\text { Facteurs } \\
\text { intrinsèques }\end{array}$} & \multicolumn{2}{|c|}{$\begin{array}{c}\text { Facteurs } \\
\text { extrinsèques }\end{array}$} & & \\
\hline & & $\mathrm{t}$ & $B$ & $\mathrm{t}$ & $B$ & $\mathrm{t}$ & $B$ & $\mathrm{t}$ & $\beta$ & $\mathrm{t}$ \\
\hline & VARIABLES SOCIODÉMOGRAPHIQUE & & & & & & & & & \\
\hline & $\begin{array}{ll}\text { Sexe } & -0,06\end{array}$ & $-0,69$ & $-0,26$ & $-2,75 * *$ & $-0,09$ & $-0,97$ & $-0,17$ & $-1,85$ & $-0,07$ & $-0,99$ \\
\hline & 0,17 & 1,90 & $-0,03$ & $-0,34$ & 0,02 & 0,20 & 0,10 & 1,12 & $-0,02$ & $-0,23$ \\
\hline & Lien avec formation $\quad 0,45$ & $4,93^{*}$ & 0,06 & 0,58 & 0,35 & $3,62 *$ & 0,20 & $2,14^{* * *}$ & 0,56 & $6,86^{*}$ \\
\hline & Statut d'emploi & 0,65 & 0,18 & 1,86 & 0,17 & 1,82 & 0,18 & 1,99 & $-0,00$ & $-0,03$ \\
\hline $\overrightarrow{8}$ & Revenu & 1,19 & 0,14 & 1,47 & 0,11 & 1,13 & 0,12 & 1,28 & 0,22 & $2,80^{* *}$ \\
\hline 2 & PERCEPTION DES FACTEURS FACILI] & ANTS & & & & & & & & \\
\hline 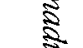 & Soutien perçu $\quad 0,11$ & 1,21 & 0,10 & 0,99 & 0,02 & 0,22 & $-0,05$ & $-0,50$ & 0,03 & 0,35 \\
\hline$\frac{i}{i}$ & R2 ajusté & 40 & & & & 31 & & 33 & & 0,19 \\
\hline 3. & $\mathrm{F}$ & $91 * *$ & 17 , & $3 * *$ & & $72 *$ & & $59 *$ & & $7,38^{*}$ \\
\hline & $B=$ standardized beta $w$ & $* * \mathrm{p}<0,0$ & $1 * *$ & 0,05 & & & & & & \\
\hline
\end{tabular}


1) plus la satisfaction face aux facteurs intrinsèques liés à l'emploi est élevée, 2) plus l'écart entre les attentes liées à l'emploi (facteurs intrinsèques et extrinsèques) et la situation réelle est perçu comme positif, et 3) plus les sujets ont l'impression d'avoir atteint leurs objectifs. Le revenu d'emploi, pour sa part, prédit uniquement le sentiment d'atteinte des objectifs, celui-ci étant d'autant plus positif que le revenu est élevé (4,1\% de variance expliquée). Finalement, la troisième variable de ce groupe, le statut de l'emploi, ne participe pas significativement à la prédiction de la satisfaction professionnelle des répondants et répondantes.

Les résultats présentés au tableau 2 expriment également le lien de prédiction existant entre la perception du caractère facilitant ou non du support social, de certains attributs personnels et de différentes caractéristiques du marché du travail sur la satisfaction professionnelle. Cette fois-ci, une seule variable, les caractéristiques perçues du marché du travail, présente des liens significatifs avec tous les indicateurs, lesquels liens s'avèrent élevés (4,3 à 30,1\% de variance expliquée). Concrètement, plus la perception qu'a le sujet du marché du travail comme facteur facilitant l'atteinte de ses objectifs est positive, et plus 1) il se montre satisfait des facteurs intrinsèques et extrinsèques de son emploi, 2) plus l'écart entre les attentes liées à l'emploi (facteurs intrinsèques et extrinsèques) et la situation réelle est perçu comme positif, et 3) plus il a l'impression d'avoir atteint ses objectifs. La perception du rôle du soutien social et de certains attributs personnels dans l'atteinte des objectifs personnels et professionnels ne parvient pas à ajouter à la prédiction de la satisfaction professionnelle.

L'effet des différentes variables indépendantes sur la variable "désir de quitter son emploi" a été évalué par une régression logistique, cette variable étant codée de façon dichotomique. La régression obtenue s'avère significative $\left(\chi^{2}(9, \mathbf{n}=72)=37,37, \mathrm{p}<0,001\right)$ et permet l'identification de trois prédicteurs, soient, les caractéristiques du marché du travail $(r=-0,30, p<0,001)$, le sexe des sujets $(r=-0,24, p<0,01)$ et le lien de l'emploi avec la formation $(r=0,22, p<0,05)$. L'équation de régression obtenue donne lieu à un taux de classement correct $(88,9 \%)$ et indique que 1$)$ plus le répondant ou la répondante perçoit que les caractéristiques du marché du travail peuvent faciliter l'atteinte de ses objectifs et plus il ou elle songe quitter son emploi, 2) les hommes ont, plus que les femmes, l'intention de quitter leur emploi et 3 ) moins le 
jeune travailleur ou la jeune travailleuse estime que son emploi s'avère en lien avec sa formation et plus il ou elle manifeste l'intention de quitter son emploi.

\section{Satisfaction personnelle}

Les régressions multiples effectuées à partir des variables indépendantes nous permettent de constater que certaines d'entre elles prédisent significativement le niveau de satisfaction personnelle lié à la possibilité de réaliser des projets personnels et au bien-être psychologique de l'individu. Le tableau 3 présente les résultats des régressions effectuées séparément sur les variables liées à la satisfaction personnelle.

Les résultats des analyses de régression effectuées sur les différentes variables liées à la satisfaction personnelle permettent de prédire jusqu'à $31 \%$ de leur variance, reflétant un niveau de prédiction moyennement satisfaisant. Parmi les variables sociodémographiques, seule la scolarité contribue à prédire un indicateur de la satisfaction personnelle, soit la possibilité de réaliser des projets personnels $(5,2 \%$ de variance expliquée). Ainsi, moins la scolarité est élevée et plus le jeune travailleur et la jeune travailleuse a le sentiment que sa situation d'emploi lui permet de réaliser des projets personnels. Ni le sexe du sujet, ni son âge n'ajoutent à la prédiction de la satisfaction personnelle.

Quant aux variables liées à l'emploi, les résultats présentés au tableau 3 montrent que deux des variables de cette catégorie permettent d'expliquer une partie de la variance de deux des indicateurs de la satisfaction personnelle (4,3 à $12,8 \%$ de variance expliquée). Plus spécifiquement, le statut de l'emploi s'avère un prédicteur du bien-être et de l'autonomie personnels, les sujets occupant un emploi à temps plein estimant que leur situation d'emploi contribue positivement à leur bien-être et à leur autonomie. Concernant la variable "revenu", elle agit comme prédicteur de la possibilité perçue de réaliser des projets personnels et du sentiment de sécurité personnelle. Ainsi, plus le revenu d'emploi est élevé et plus le jeune travailleur ou la jeunes travailleuse considère que sa situation d'emploi affecte positivement la réalisation de ses projets personnels de même que son sentiment de sécurité personnelle. Enfin, notons que la variable "lien de l'emploi avec la formation" ne s'avère pas un prédicteur significatif de la satisfaction personnelle. 


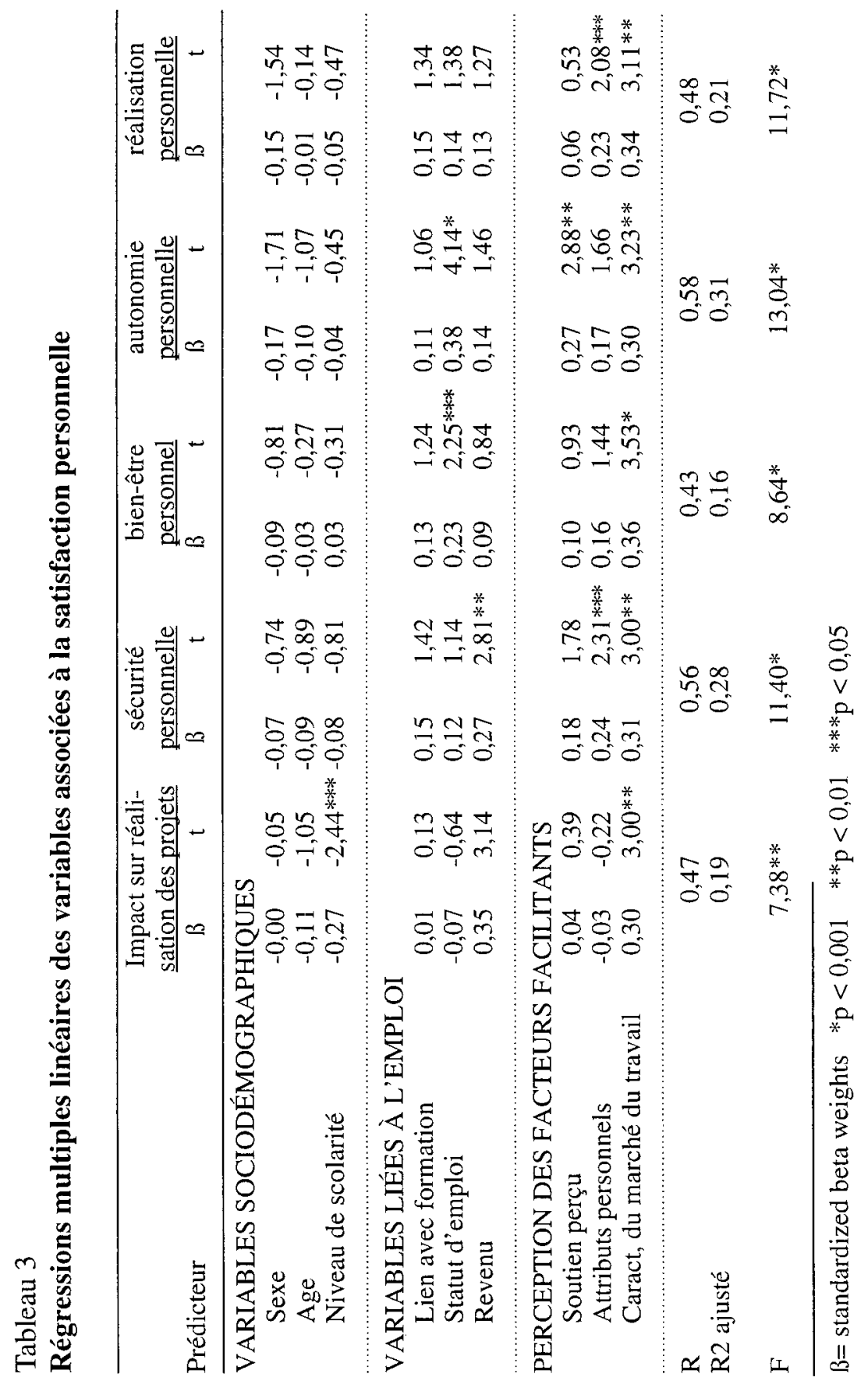

The Canadian Journal of Higher Education

Volume XXIX, No. 1, 1999 
Finalement, tel que l'indiquent les résultats présentés au tableau 3, les variables les plus prédictives de la satisfaction personnelle sont d'ordre perceptuel. La variable "soutien social perçu" permet de prédire une partie de la variance de l'autonomie personnelle $(6,4 \%$ de la variance expliquée). Concrètement, plus le sujet perçoit que le soutien familial facilite l'atteinte de ses objectifs et plus il considère que sa situation d'emploi actuelle favorise son sentiment d'autonomie personnelle.

Les attributs personnels, quant à eux, contribuent à la prédiction du sentiment de sécurité et de réalisation personnels (environ 3,3 à 4,1\% de la variance expliquée). Ainsi, plus l'individu perçoit que ce sont ses propres attributs qui favorisent l'atteinte de ses objectifs et plus il aura tendance à évaluer positivement ses sentiments de sécurité et de réalisation personnelles.

Finalement, la variable «caractéristiques perçues du marché du travail" s'avère un prédicteur significatif de l'ensemble des cinq indicateurs de la satisfaction personnelle $(9,0$ à $17,8 \%$ de variance expliquée). Les résultats présentés au tableau 3 indiquent que plus les sujets perçoivent que ce sont les caractéristiques du marché du travail qui favorisent l'atteinte de leurs objectifs, plus ils estiment que leur situation d'emploi actuelle influence positivement la réalisation de leurs projets de vie de même que leurs sentiments de sécurité, de bien-être, d'autonomie et de réalisation personnels. Notons que le cinquième indicateur de la variable "satisfaction personnelle", soit le degré de stress ressenti par le jeune travailleur ou la jeune travailleuse, ne s'avère significativement corrélé à aucune des variables indépendantes de l'étude.

\section{Sentiment de contrôle de la situation financière}

Les régressions multiples effectuées à partir des variables indépendantes nous permettent de constater que certaines d'entre elles prédisent significativement le sentiment de contrôle de la situation financière (satisfaction générale face à la situation financière, perception de l'équilibre financier et précarité perçue). Le tableau 4 présente les résultats des régressions effectuées séparément sur ces trois indicateurs liés au sentiment de contrôle de la situation financière.

Tel que le présentent les résultats du tableau 4, les variables indépendantes permettent de prédire jusqu'à $25 \%$ de la variance liée au sentiment de contrôle de la situation financière, témoignant d'une capacité pronostique pouvant atteindre un niveau moyennement satisfaisant. 


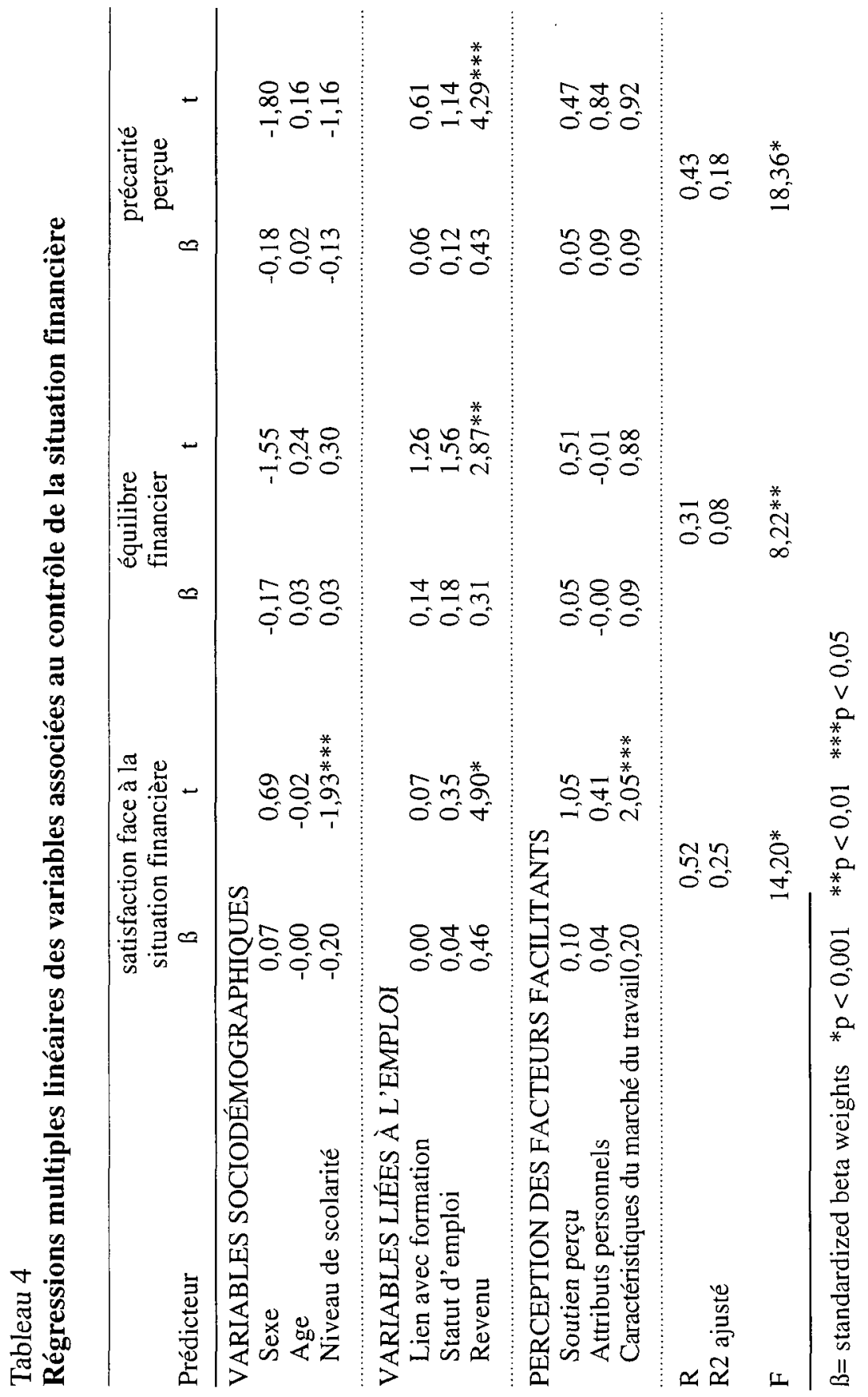

The Canadian Journal of Higher Education

Volume XXXX, No. 1, 1999 
De façon plus précise, les résultats présentés au tableau 4 indiquent que parmi les variables sociodémographiques, seul le niveau de scolarité parvient à prédire un des indicateurs du sentiment de contrôle de la situation financière, soit la satisfaction face à la situation financière (environ $4 \%$ de la variance expliquée). Ainsi, plus le jeune travailleur ou la jeune travailleuse est scolarisé-e et plus il ou elle se dit satisfait-e de sa situation financière.

Pour leur part, les variables liées à l'emploi prédisent, uniquement par le biais du revenu toutefois, les trois indicateurs associés au sentiment de contrôle de la situation financière (de 8,2 à $21,7 \%$ de la variance expliquée). Concrètement, plus le revenu d'emploi du jeune travailleur ou de la jeune travailleuse est élevé, plus des derniers se sentent privilégiés de leur situation financière, plus ils estiment que leurs obligations financières sont inférieures à leurs revenus et moins ils considèrent leur situation d'emploi comme étant précaire.

Enfin, concernant la perception des facteurs facilitants, seule la variable "caractéristiques perçues du marché du travail" contribue à prédire l'un des trois indicateurs du sentiment de contrôle de la situation financière, soit le sentiment de sécurité face à la situation financière (3,1\% de la variance expliquée). Ainsi, plus les sujets perçoivent que les caractéristiques du marché du travail favorisent l'atteinte de leurs objectifs et moins ils se sentent inquiets de leur situation financière.

Pour conclure cette section, ajoutons quelques résultats complémentaires qui permettent de nuancer les résultats présentés aux tableaux 2,3 et 4 . Ces résultats sont tirés d'analyses de corrélation bivariées et mettent en évidence la contribution prépondérante des variables perceptuelles et de celles liées à l'emploi dans la compréhension de la qualité de l'insertion socioprofessionnelle des diplômés. Les relations établies atteignent ou dépassent le seuil de $\mathrm{r}=0,30$, généralement associé à une corrélation moyenne (Meltzoff, 1998), pour environ $75 \%$ des corrélations significatives impliquant l'une ou l'autre de ces quatre variables. De l'angle des variables dépendantes, par ailleurs, les résultats indiquent que les variables "satisfaction professionnelle" et "satisfaction personnelle" s'avèrent mieux prédites que ne l'est la variable "sentiment de contrôle de la situation financière", en ce qu'elles se trouvent plus fortement corrélées avec les différentes variables indépendantes. En outre, parmi les variables perceptuelles, notons la contribution particulière de la variable "rôle perçu des attributs 
personnels". Si cette dernière ne s'est avérée un prédicteur significatif dans aucune des régressions multiples, des analyses de corrélation bivariées ont permis de constater qu'elle est pourtant significativement reliée à 11 des indicateurs de la qualité de l'insertion socioprofessionnelle $(r=0,22$ à $r=0,39, p<0,05)$.

\section{DISCUSSION}

L'objectif poursuivi dans le présent article était de présenter un schéma exploratoire regroupant trois catégories de variables servant à définir la qualité de l'insertion socioprofessionnelle de nouveaux travailleurs et travailleuses diplômés et trois autres catégories de variables susceptibles de prédire la qualité de cette insertion. Ce schéma a été mis à l'épreuve auprès d'un échantillon constitué de nouveaux travailleurs et travailleuses, diplômés depuis trois ans. Certaines données descriptives ont d'abord été présentées pour éclairer la qualité de la situation d'insertion socioprofessionnelle de ces sujets: est-ce que les nouveaux travailleurs et travailleuses diplômés sont satisfaits de leur emploi? Ce dernier répond-il à leurs attentes? Leur situation d'emploi leur permet-elle d'élaborer des projets personnels et d'améliorer leur qualité de vie? Trois ans après avoir terminé leurs études, se sentent-ils maintenant en contrôle de leur situation financière et en sécurité face à celle-ci ?, sont quelques-unes des questions auxquelles nous avons tenté de répondre dans cette première partie. Ensuite, les résultats des analyses de régression ont permis de mieux saisir les liens existant entre les différentes composantes du schéma exploratoire. En effet, ces analyses ont mis en lumière la force relative de certains critères objectifs comme le sexe du sujet et le statut de l'emploi et celle de critères plus subjectifs comme la perception des facteurs facilitants, dans la prédiction de la qualité de l'insertion vécue par de jeunes adultes trois ans après l'obtention de leur diplôme.

Si l'ensemble des résultats présentés au tableau 1 laissent entrevoir un niveau de satisfaction relativement acceptable tant au niveau professionnel, personnel, que financier, il n'en demeure pas moins que le fait qu'il s'agisse des seuls diplômés de l'échantillon global qui soient actuellement en emploi, vient relativiser l'interprétation que l'on peut faire de ces données. En effet, si les résultats indiquent un taux de satisfaction générale assez bon en regard de l'emploi, il n'en demeure pas moins qu'environ $30 \%$ des sujets, trois ans après leur entrée sur le 
marché du travail, estiment toujours qu'ils n'ont pas atteint leurs objectifs et que leur situation professionnelle affecte négativement la mise en oeuvre de projets de vie importants. Il est probable que ce résultat soit lié au fait que près du tiers d'entre eux expriment, qui des inquiétudes, ou qui du découragement, à l'égard de leur situation financière. En fait, on constate que si ces nouveaux travailleurs et travailleuses semblent généralement assez heureux en emploi, ils se sentent toutefois relativement pauvres et retardent certains projets personnels à moyen et à long termes. Ainsi, on peut faire l'hypothèse que le contexte économique difficile que nous connaissons actuellement affecte d'abord et avant tout la qualité de vie des jeunes et leur entrée dans la vie adulte plutôt que strictement leur bien-être en emploi ou leur rapport au marché du travail. En effet, si d'un côté, ils ne sont pas réellement insatisfaits ou inquiets de leur situation, d'un autre côté, ils ne fournissent pas d'indices clairs que leur emploi ou leur situation sur le marché du travail leur a réellement donné accès aux libertés et aux possibilités qui ont longtemps été associées à — sinon garanties par l'obtention d'un emploi.

Par ailleurs, les différences observées selon le niveau de scolarité des sujets font ressortir que, pour au moins une des variables de chacun des groupes liés à la satisfaction professionnelle, personnelle ou de contrôle de la situation financière, les étudiants de niveau collégial témoignent d'une qualité d'insertion significativement moindre que celle de leurs collègues du secondaire et de l'université. Plus précisément, ces sujets de niveau collégial disent se sentir peu reconnus, peu valorisés dans leur emploi et y perçoivent moins de possibilités de réalisation personnelle qu'ils n'avaient anticipé. Ils expriment en outre un sentiment plus prononcé de précarité financière que les sujets du secondaire ou de l'université. En fait, il semble clair que ce ne sont pas les conditions d'emploi, ni les facteurs extrinsèques à l'emploi qui amoindrissent la satisfaction de ce groupe mais plutôt certains indicateurs liés à l'identité et à l'épanouissement personnels, lesquels ont peut-être été associés plus étroitement, par ce groupe de jeunes, à l'obtention d'un emploi. Trois hypothèses peuvent être posées en regard de ce résultat. La première concerne la possibilité que plusieurs programmes de technique collégiale forment des travailleurs trop qualifiés pour les exigences des emplois qui leur sont habituellement destinés, situation très peu propice à l'affirmation de l'identité personnelle et professionnelle. La deuxième 
hypothèse renvoie au fait que les postes généralement obtenus par les diplômés de technique collégiale, même si en lien avec leur formation, exigent bien souvent l'exécution de tâches "connexes", qui font peu appel à leur expertise et favorisent très peu, du même coup, le développement de nouvelles compétences et la valorisation personnelle. Finalement, une troisième hypothèse concerne cette fois les attentes particulières qui pourraient être nourries par plusieurs diplômés de technique collégiale. Il est possible en effet qu'ayant décidé de dépasser le cap déterminant du diplôme secondaire (OCDE, 1996), ces jeunes aient développé, face à leur futur emploi, des attentes et des aspirations personnelles allant bien au-delà des degrés d'autonomie, de renforcement et de développement qu'offrent souvent les emplois de nature technique. Cette dernière hypothèse trouve d'ailleurs un certain appui dans un récent article de Buckley, Fedor, Veres, Wiese et Carraher (1998), lequel exprime clairement que le fait d'entretenir des attentes élevées vis-à-vis du travail augmente la probabilité d'être insatisfait en emploi. Ces auteurs suggèrent même la mise en place de programmes visant à amoindrir les attentes des nouveaux travailleurs et travailleuses de façon à ajuster celles-ci à la réalité du marché du travail et à éviter de la sorte ce qu'ils appellent "the reality shock".

Finalement, contrairement aux résultats de nombreuses études, très peu de différences ont été observées entre les hommes et les femmes pour l'ensemble des variables proposées dans le schéma exploratoire. La seule différence observée a trait au niveau significativement plus élevé de précarité financière ressenti par les femmes. Ce peu de différences peut être expliqué par le fait que l'écart dans la façon dont les hommes et les femmes expérimentent le travail se manifeste généralement plus tard dans la vie professionnelle, particulièrement lorsque les femmes sont appelées à concilier leur vie familiale et professionnelle (Fahmy, 1995).

Concernant la prédiction de la qualité de l'insertion socioprofessionnelle, un premier regard jeté sur les résultats des tableaux 2, 3 et 4 permet de constater que toutes les variables indépendantes, sauf l'âge, contribuent à comprendre les différentes facettes exprimant la qualité de l'insertion des jeunes travailleurs et travailleuses. Cette première constatation vient confirmer la pertinence des relations proposées dans le schéma exploratoire. Certaines de ces variables contribuent à la prédiction de façon marginale et d'autres, de façon nettement plus récurrente. Par contre, des corrélations bivariées 
établissent des liens plus évidents et plus stables entre les différentes variables du schéma. De plus, aucun des trois groupes de variables dépendantes ne peut être mis de côté sans que la compréhension de la qualité globale de l'insertion perde une facette importante et unique. En effet, tel que proposé dans le schéma exploratoire, une insertion de qualité dépasse largement la satisfaction professionnelle de même qu'elle ne peut se limiter à la satisfaction personnelle retirée de la situation d'emploi ou au sentiment de contrôle de la situation financière qui en découle. Plus concrètement, selon le schéma exploratoire proposé, le seul fait qu'il manifeste une satisfaction moyenne ou positive en regard de son emploi ne permet pas de conclure qu'un jeune travailleur ou une jeune travailleuse éprouve une insertion socioprofessionnelle globale de qualité. De la même manière, le fait de se sentir en sécurité financièrement, critère souvent utilisé pour traduire l'insertion complétée, ne peut davantage traduire adéquatement l'expérience globale d'insertion du jeune travailleur ou de la jeune travailleuse.

De manière plus spécifique, trois constatations se dégagent des analyses de régression, soit le peu de valeur pronostique des variables sociodémographiques sur la qualité de l'insertion; la prépondérance marquée de la variable "caractéristiques perçues du marché du travail" pour prédire la qualité de l'insertion et, finalement, l'importance prédictive du lien de l'emploi avec la formation.

\section{Valeur pronostique des variables sociodémographiques}

Tout d'abord, et contrairement à ce qui est généralement observé dans les différentes études, la qualité de l'insertion des jeunes travailleurs et travailleuses n'a pu être prédite de façon stable par le sexe, l'âge et le niveau de scolarité des sujets. Cependant, il faut mentionner que les études recensées ont généralement pour but de vérifier la valeur de ces variables dans la prédiction de l'obtention d'un emploi après les études, de la qualité de l'emploi obtenu ou de la satisfaction face à celui-ci (Bengle, 1991; Tanguay, 1986; Touzard et al.,1996; Trottier et al., 1995). Ainsi, il est possible de croire que l'impact des variables sociodémographiques se fait ressentir plus intensément immédiatement à la sortie du système éducatif et que l'effet de cohorte tend à s'estomper quelques années après l'obtention du diplôme pour laisser davantage de place à l'influence des variables d'ordre perceptuel. Ainsi, plus on raffine la définition de la qualité de 
l'insertion et qu'on l'étudie sous des angles plus subjectifs, plus on constate que certaines différences liées à la personne elle-même et à sa situation d'emploi s'avèrent davantage déterminantes, au fil des années, de la qualité globale de l'insertion socioprofessionnelle des sujets que ne peut l'être l'appartenance à l'une ou l'autre des cohortes. En somme, il appert que plus on s'éloigne de l'étape de la diplômation et moins le cheminement professionnel ne s'avère déterminé par des caractéristiques fixes, sur lesquelles l'individu a peu ou pas de contrôle.

\section{Prépondérance marquée de la variable "caractéristiques perçues du marché du travail" pour prédire la qualité de l'insertion}

Parmi toutes les variables proposées dans le schéma, la perception de caractéristiques facilitantes du marché du travail dans l'atteinte des objectifs s'est révélée être le plus puissant prédicteur de la qualité de l'insertion socioprofessionnelle des jeunes. Tei qu'évoqué dans la présentation du schéma exploratoire, ce résultat indique, d'une part, que ce ne sont pas uniquement les variables structurelles de l'emploi qui déterminent si le sujet se sent satisfait de sa situation générale d'insertion mais aussi sa manière subjective d'être en relation avec le marché du travail. D'autre part, ils signifient aussi que parmi les variables perceptuelles, celles qui sont liées au support social ou aux caractéristiques personnelles ont moins de poids relatif dans la prédiction que celles qui sont liées au marché du travail. En fait, nos résultats indiquent que la qualité de l'insertion est passablement tributaire des caractéristiques perçues du marché du travail, mettant dès lors en évidence les limites d'une conception de la réussite de l'insertion strictement liée à l'obtention d'un emploi à temps plein et d'un revenu élevé. On peut penser que la manière de vivre les premières expériences en emploi dépend largement de l'impression qu'a le sujet de pouvoir tirer profit de certaines caractéristiques du marché du travail, lesquelles, dans un contexte économique n'offrant guère de soutien, peuvent souvent être perçues comme des obstacles plutôt que comme des facteurs facilitants. Plus concrètement, un individu dont le revenu serait plutôt faible mais qui aurait néanmoins l'impression que le marché du travail est porteur d'opportunités et de conditions facilitantes pourrait afficher un niveau de satisfaction professionnelle et personnelle supérieur à celui d'un autre dont le revenu serait plus élevé mais qui 
croirait plutôt que le marché du travail est jonché d'obstacles à l'atteinte de ses objectifs.

Pour ce qui est de la prépondérance de la variable "caractéristiques perçues du marché du travail" sur les deux autres variables perceptuelles, une nuance s'impose. En effet, comme l'ont indiqué les résultats des analyses bivariées, la variable "rôle perçu des attributs personnels" se trouve reliée de façon significative et importante à la majorité des variables liées à la qualité de l'insertion des individus (11 sur 14). Ces résultats viennent relativiser la place des caractéristiques du marché à titre de prédicteur et suggèrent que, dans un contexte où les opportunités du marché du travail seraient plus fréquentes ou avantageuses, le rôle des attributs personnels dans l'atteinte des objectifs pourrait s'avérer plus déterminant qu'il ne l'est ici (tant en terme relatif qu'absolu) dans la prédiction de la qualité de l'insertion. De la même façon, mais de manière plus restreinte toutefois, les résultats des analyses bivariées ont également permis d'observer un lien significatif entre le rôle du support social perçu et certaines variables liées à la qualité de l'insertion (4 sur 14). Encore une fois, on peut croire que dans un contexte d'emploi plus facilitant, le rôle de cette variable dans la manière positive ou négative de vivre les premières années sur le marché du travail pourrait apparaître avec plus d'évidence.

\section{Importance prédictive du lien de l'emploi avec la formation sur la qualité de l'insertion}

Parmi les variables liées à l'emploi, la variable la plus subjective, celle du lien de l'emploi avec la formation, s'est avérée plus prédictive de la qualité de l'insertion que le revenu du sujet et même du statut de son emploi. Ce résultat suggère que la possibilité qu'ont les jeunes d'agir en fonction de leurs projets et des objectifs qu'ils se sont fixés en s'inscrivant à tel ou tel programme d'études est déterminante de la satisfaction professionnelle et personnelle expérimentée au cours de leurs premières années sur le marché du travail. Encore une fois, ce résultat renvoie à l'importance de considérer les intérêts du sujet, ses projets, et ses choix dans la compréhension de l'insertion socioprofessionnelle, tout autant que les variables plus structurelles comme le revenu de l'emploi ou son statut. On peut ainsi faire l'hypothèse que travailler dans un secteur qu'ils ont choisi, est, pour les jeunes, davantage garant de leur satisfaction qu'un revenu élevé ou un 
poste à temps plein, suggérant dès lors que la réalisation personnelle au travail demeure, malgré le contexte difficile, fort importante pour les nouveaux travailleurs et travailleuses.

Pour conclure, le schéma exploratoire présenté ici regroupe et élargit plusieurs des cadres généralement utilisés pour traiter de la question de l'insertion et, de ce fait, ajoute aux éléments qui doivent être considérés pour comprendre la qualité du cheminement professionnel et l'aboutissement du processus d'insertion socioprofessionnelle. De fait, le schéma proposé est novateur à au moins deux égards. D'abord, il propose de recourir, en plus des variables habituelles (sociodémographiques et liées à l'emploi), à des variables perceptuelles pour prédire la qualité de l'insertion socioprofessionnelle de diplômés. Ensuite, il vient raffiner la conception de la qualité de l'insertion en en proposant une perspective à facettes multiples et en cherchant à identifier les processus qui lui sont sous-jacents. En effet, bien souvent les tentatives pour investiguer la qualité de l'insertion s'attardent davantage à un ou deux des trois groupes de variables du schéma exploratoire et ne tiennent pas toujours suffisamment compte des nombreuses interactions existant entre les sphères professionnelles et personnelles, interactions particulièrement importantes chez les jeunes adultes dont l'avenir est en construction. D'autres études sont nécessaires pour valider ce schéma exploratoire, pour étudier plus en détails' la nature et l'ampleur des interrelations entre les différentes variables de même que pour en vérifier les applications possibles à d'autres étapes de la carrière.

\section{Références}

Bengle, N.M. (1991). L'insertion professionnelle des jeunes: une étude des processus socio-psychologiques dans le secteur des services, [Thèse]. SteFoy, PQ: Université Laval.

Bruhn, J.G. (1989). Job stress: An opportunity for professional growth. Career Development Quarterly, 37(4), 306-315.

Buckley, R.M., Fedor, D.B., Veres, J.G.,Wiese, D.S., \& Carraher, S.M. (1998). Investigating newcomer expectations and job-related outcomes, 83(3), 452-461. 
Cairns, K., Woodward, J.B., \& Hashizume, L.G. (1992). Employment counselors' and youth's views of the transition to work: Preparing to develop a work skills simulation. Canadian Journal of Counselling, 26(4), 222-239.

Conseil Permanent de la Jeunesse. (1997). La réforme de la sécurité du revenu. Un parcours semé d'embûches. Québec, PQ Gouvernement du Québec.

Coye, J.C., \& Bolger, N. (1990). Doing without social support as an explanatory concept. Journal of Social and Clinical Psychology, 9(1), 148-158.

Coyne, J.C., \& De Longis, A. (1986). Going beyond social support: The role of social relationships in adaptation. Journal of Consulting and Clinical Psychology, 54(4), 454-460.

Curie J. (1993). Faire face au chômage. L'Orientation scolaire et professionnelle (numéro spécial), 22(4), 295-303.

Fahmy, P. (1995). Femmes: entre vie et carrière. Québec, PQ: Éditions Adage.

Fournier, G, \& Bellerive, A. (1991). L'insertion socio-professionnelle des étudiants universitaires: les mythes et les réalités. Actes du XIVe Congrès Mondial de l'Association Internationale d'Orientation Scolaire et Professionnelle, Montréal, PQ, 173-180.

Fournier, G., \& Croteau, L. (1997). L'insertion socioprofessionnelle: Une réalité qui dépasse l'obtention d'un emploi. Communication présentée dans le cadre de l'ACFAS. Trois-Rivières, $\mathrm{PQ}$.

Galland, O. (1995). Une entrée de plus en plus tardive dans la vie active. Economie et Statistique, 283-284, 35-52.

Gauthier, M. (1992). Des travailleurs en attente d'emploi, In D.-G. Tremblay (ed.), Travail et société. Une introduction à la sociologie du travail, pp. 521-543. Laval, PQ: Éditions Agence d'Arc.

Gauthier, M. (1994). Une société sans les jeunes? Québec, PQ: Institut québécois de recherche sur la culture.

Hartnagel, T.F., \& Krahn, H. (1995). Labour market problems, and psychological well-being: A panel study of canadian youth in transition from school to work. British Journal of education and work, 8(3), 33-53.

Kabanoff, B. (1982). Psychological effects of unemployment: a consideration of some alternative explanation. Australian and New Zealand Journal of Psychiatry, 16, 37-42.

Matte, D. Baldino, D., \& Courchesne, R.(1998). L'évolution de l'emploi atypique. Québec, PQ: Les Publications du Québec, Ministère du Travail.

Meltzoff, J. (1998). Critical thinking about research. Washington, DC: American Psycholocial Association.

Moreau, N. (1997). Examen de certaines dimensions de l'insertion professionnelle liées au marché du travail. Sainte-Foy,m PQ: Conseil Supérieur de l'Éducation. 
OCDE (1996). Transition de la formation initiale à la vie active. Examen thématique: domaine, objectifs, cadre analytique et procédures de mise en oeuvre, Paris: OCDE, 1-29.

Pierce, G.R., Sarason, B.R., \& Sarason, I.G. (1991). General and relationshipbased perceptions of social support: Are two constructs better than one? Journal of Personality and Social Psychology, 61(6), 1028-1039.

Rascle, N. (1994). Le soutien social dans la relation stress-maladie. In M. Bruchon-Schweitzer, \& R. Dantzer (éd.), Introduction à la psychologie de la santé, (pp. 125-153). Paris: Presses universitaires de France.

Sarason, B.R., Pierce, G.R., \& Sarason, I.G. (1990). Social support: the sense of acceptance and the role of relationships. In B.R. Sarason, I.G. Sarason \& G.R. Pierce (ed.), Social support: an interactional view. New York, NY: Wiley.

Tanguy, L. (1986). L'introuvable relation formation-emploi. Paris: La Documentation Française.

Touzard, H., Lancry-Hoestlandt, A., Depolo, M., \& Sarchielli, G. (1996). Dynamique de la satisfaction au travail chez les jeunes dans leur premier emploi. Étude longitudinale internationale. Psychologie du travail et des organisations, 2(1-2), 26-42.

Tremblay, D.-G. (1994). Chômage, flexibilité et précarité d'emploi: aspects sociaux. In F. Dumont, S. Langlois, \& Y. Martin (éd.), Traité des problèmes sociaux, (pp. 623-652). Québec, PQ: Institut québécois de recherche sur la culture.

Trottier, C., Perron, M., \& Diambomba, M. (1995). Les cheminements scolaires et l'insertion professionnelle des étudiants de l'Université. Laval, PQ: Les Presses de l'Université Laval.

Vincens, J. (1996). L'insertion professionnelle des jeunes. Délimiter un champ de recherche? Laboratoire interdisciplinaire de recherche sur les Ressources Humaines et l'Emploi (LIRHE), Unité de recherche associée au CNRS, Université des Sciences Sociales, Toulouse. 1-50.

Vincens, J. (1998). L'insertion professionnelle des jeunes. À la recherche d'une définition conventionnelle. Formation emploi, 60, 21-36. 